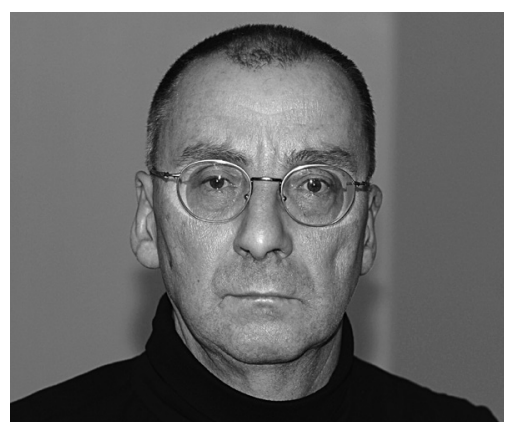

\section{Mittler zwischen Staat, Markt und Zivilgesellschaft}

»Kooperationen bei der Leistungserbringung « - das Titelthema dieser Ausgabe mag theoretisch klingen, hat aber eminent praktische Konsequenzen für alle Beteiligten: Die Sozialleistungsträger müssen ihre gesetzlichen Pflichten kennen und wahrnehmen, die Leistungserbringer haben den Spagat zwischen knappen Mitteln und Angeboten zu bewältigen, die Nutzer und ihr Umfeld müssen ihre eigenen Möglichkeiten einsetzen. Und dieses Zusammenspiel muss - über den Einzelfall hinaus - koordiniert werden und dauerhaft funktionieren. Unternehmen der Sozialwirtschaft werden in diesem Zusammenhang neuerdings als »hybride Organisationen« betrachtet, wie Andreas Strunk in seinem Beitrag beschreibt. Gemeint sei damit der Umstand, dass diese Unternehmen einen schwierigen Balanceakt zu bewältigen hätten durch die Platzierung bedarfsgerechter Angebote im Spannungsfeld von Staat, Markt und Zivilgesellschaft. Öffentliche Sozialplanung stehe dabei vor einer ganz ähnlichen Aufgabe. Sie muss Aussagen machen über gelingendes Leben von Leistungsberechtigten im konkreten Lebens- und Sozialraum der betroffenen Menschen. In diesem befänden sich glücklicherweise nicht nur Anbieter von Leistungen, die nach unterschiedlichen Sozialgesetzbüchern finanziert würden. Es gehe zunehmend auch um das Aufspüren komplementärer Ressourcen. Die Forderung nach Sozialraumorientierung habe dann erhebliche Konsequenzen auf die Gestaltung von Kooperationen im Bereich der Leistungserbringung.

Über mögliche Alternativen zum gegenwärtigen Wirtschaften geht es auch beim nächsten Kongress der Sozialwirtschaft vom 12. bis 14. Juni 2013 in Magdeburg. Der österreichische Wirtschaftsexperte und Autor Christian Felber wird in diesem Branchentreff sein von ihm mitentwickeltes Modell der »Gemeinwohl-Ökonomie « vorstellen, in dem der Unternehmensgewinn am Nutzen für die Allgemeinheit gemessen wird. Aktuelle Informationen zu dem Kongress gibt es auf einer eigenen Website: www.sozkon.de. 